\title{
Strahleninduziertes kutanes Angiosarkom mit atypischem Metastasierungsmuster
}

\section{Unusual Metastatic Pattern of a Radiation Induced Angiosarcoma of the Skin}

\author{
Autoren \\ S. Hege ${ }^{1}$, J. Knolle', J. Schreiber ${ }^{2}$ \\ Institute \\ Institut für Pathologie, Städtisches Klinikum Dessau \\ Fachbereich für Pneumologie, Otto-von-Guericke-Universität Magdeburg
}

\section{Bibliografie}

DOI $10.1055 / \mathrm{s}-2008-1077538$

Online-Publikation:

15. 10.2008

Akt Dermatol 2008; 34:

497-500 @ Georg Thieme

Verlag KG Stuttgart · New York

ISSN 0340-2541

Korrespondenzadresse

Stefanie Hege

Institut für Pathologie

Städtisches Klinikum Dessau

Auenweg 38

06847 Dessau

stefanie.hege@

klinikum-dessau.de

\section{Zusammenfassung \\ $\nabla$}

Wir berichten über eine zum Aufnahmezeitpunkt 67-jährige Patientin, bei der 27 Jahre zuvor eine Hysterektomie und Oophorektomie mit anschließender Telekobaltbestrahlung bei einem Ovarialkarzinom durchgeführt wurde. 25 Jahre danach entwickelten sich multiple Knoten im ehemaligen Bestrahlungsgebiet in der Bauchwand. Die histologischen Untersuchungen zeigten eine epitheloide Neubildung mit vaskulärer Proliferation und einem feinen Netzwerk retikulärer Fasern, die Immunhistochemie eine angiogene Differenzierung. Es wurde ein Angiosarkom diagnostiziert und eine chirurgische Therapie durchgeführt. Nach weiteren zwei Jahren stellte sich die Patientin mit schwerer Dyspnoe vor. Klinisch imponierten ausgeprägte Tumorknoten im Bereich der Bauchdecke. Im Röntgen und in der Computertomografie der Thoraxorgane fanden sich ausgeprägte, überwiegend interstitielle Infiltrate in

\section{Einleitung}

$\nabla$

Sarkome sind vergleichsweise seltene maligne Tumorentitäten. Sie können als klein-, rund- und blauzellige Tumoren imponieren und damit eine breite Differenzialdiagnose erfordern [1-3]. Die aktinische Genese von Malignomen ist ein bekanntes Phänomen [4-9], die Induktion eines Angiosarkoms infolge einer Strahlentherapie hingegen, ist eine extreme Seltenheit [1,10-13].

Metastasen von Karzinomen oder Sarkomen imponieren meist als noduläre Strukturen. Atypische Metastasierungsmuster können andere Erkrankungen imitieren und eine differenzialdiag-

${ }^{*}$ Nach einem Vortrag gehalten anlässlich des Symposions „Moderne Aspekte der Dermatoonkologie“ organisiert vom Tumorzentrum und der Klinik für Dermatologie, Venerologie und Allergologie - Immunologisches Zentrum, Städtisches Klinikum Dessau, März 2007. beiden Lungen. Die Bronchoskopie zeigte eine diffus verdickte Bronchialmukosa. In der bronchoalveolären Lavage (BAL), in Bronchialschleimhautbiopsien und transbronchialen Lungenbiopsien wurde ein maligner klein-, rund- und blauzelliger Tumor und immunhistochemisch eine Metastasierung des Angiosarkoms nachgewiesen. Die Patientin verstarb an dem fortgeschrittenen Tumorleiden. Die Obduktion bestätigte Rezidive des Angiosarkoms in der Bauchwand und bronchopulmonale Metastasen, welche ein überwiegend interstitielles und peribronchiales Wachstumsmuster aufwiesen. Weiterhin waren Metastasen in Lymphknoten, im Myokard, im abdominellen Fettgewebe und in einem synchronen Nierenzellkarzinom nachweisbar. Radiogen induzierte Angiosarkome sind sehr selten. Das Metastasierungsmuster mit dem klinischen Bild einer interstitiellen Lungenerkrankung sowie die Metastase in einem zweiten, unabhängigen, malignen Tumor ist eine extreme Rarität.

nostische Herausforderung darstellen.

Wir stellen den Fall einer Patientin mit einem primären kutanen Angiosarkom nach adjuvanter Strahlentherapie bei kurativ operiertem Ovarialkarzinom vor, das im weiteren Verlauf ein ungewöhnliches Metastasierungsmuster aufwies. Die differenzialdiagnostischen Kriterien klein-, rundund blauzelliger Infiltrate und Tumoren werden beschrieben.

\section{Fallvorstellung \\ $\nabla$}

\section{Anamnese}

1977 erfolgte bei der zum Aufnahmezeitpunkt 67-jährigen Patientin eine Hysterektomie und Oophorektomie bei einem Ovarialkarzinom mit konsekutiver Telekobaltbestrahlung des Operationsgebiets. 
Im Oktober 2002, nach 25 Jahren, entwickelten sich multiple rötliche Knoten in der Bauchdecke. Es erfolgte eine mehrzeitige Resektion, der Befund konnte jedoch nicht im Gesunden entfernt werden.

Die histologischen Untersuchungen zeigten eine maligne epitheloide Neubildung mit starker Vaskularisation ( $\bullet$ Abb. 1 a). In der Silberimprägnation nach Gomori stellte sich ein dichtes Retikulinfasernetz dar. Immunhistochemisch reagierten die Tumorzellen positiv mit Antikörpern gegen Vimentin, CD31 sowie fokal CD34 ( Abb.1b). Panzytokeratin, Zytokeratin 8/18, CA125 und CD45 hingegen waren negativ.

Somit konnte eine maligne angiogene Neoplasie der Haut - ein Angiosarkom - im ehemaligen Strahlengebiet diagnostiziert werden.

\section{Aufnahmebefund}

Im Juli 2004 stellte sich die zu diesem Zeitpunkt 67-jährige Patientin mit neu aufgetretener schwerer Luftnot in Ruhe und bei minimaler körperlicher Belastung sowie einer produktiven Hustensymptomatik mit Expektoration von sehr zähem Sekret in der Klinik vor.

\section{Diagnostische Untersuchungen}

Eine Röntgenuntersuchung und eine Computertomografie der Thoraxorgane zeigte eine ausgeprägte diffuse, vorwiegend interstitielle Zeichnungsvermehrung des Lungenparenchyms.

Unter dem klinischen und röntgenologischen Bild einer interstitiellen Lungenerkrankung erfolgte eine Bronchoskopie mit bronchoalveolärer Lavage (BAL) und Biopsien (Schleimhaut- und transbronchiale Biopsien). Die BAL zeigte lichtmikroskopisch 90\% Lymphozyten und 10\% Makrophagen. Differenzialdiagnostisch wurden ein Lymphom und eine exogen-allergische Alveolitis erörtert.

In der immunzytologischen Untersuchung waren die lymphoiden Zellen CD45 negativ, CD31 hingegen kräftig positiv, was eine angiogene Differenzierung belegte und Lymphozyten ausschloss.

Sowohl in den Schleimhautbiopsien, als auch in den transbronchialen Lungenbiopsien waren dichte Infiltrate durch lymphozytenähnliche Zellen nachweisbar ( $\bullet$ Abb.2a). Weiterhin stellte sich eine starke Vaskularisation dar, sodass die vorläufige Diagnose maligner klein-, rund- und blauzelliger Tumor war und aufgrund des Baumusters eine angiogene Neubildung diskutiert wurde. Immunhistologisch reagierten die Tumorzellen mit Antikörpern gegen CD45 und Zytokeratin negativ, mit Antikörpern gegen CD31 jedoch kräftig positiv ( $\mathbf{A b b} \mathbf{2} \mathbf{2}$ ), sodass die abschließende Diagnose bronchopulmonale Metastasierung des kutanen Angiosarkoms lautete.

\section{Verlauf}

Im August 2004 verstarb die Patientin trotz Einleitung einer zytostatischen Therapie an den Folgen der ausgedehnten Metastasierung des Angiosarkoms.

\section{Obduktionsbefund}

Es stellten sich die bekannten multiplen rötlichen Tumorknoten im Narbenbereich der Bauchdecke dar ( $\bullet$ Abb. 3).

Weiterhin waren eine massive diffuse peribronchiale und interstitielle pulmonale Metastasierung, multiple abdominelle, inguinale und thorakale lymphonodale Metastasen mit Ausbildung von Lymphknotenkonglomeraten retroperitoneal, Metas-

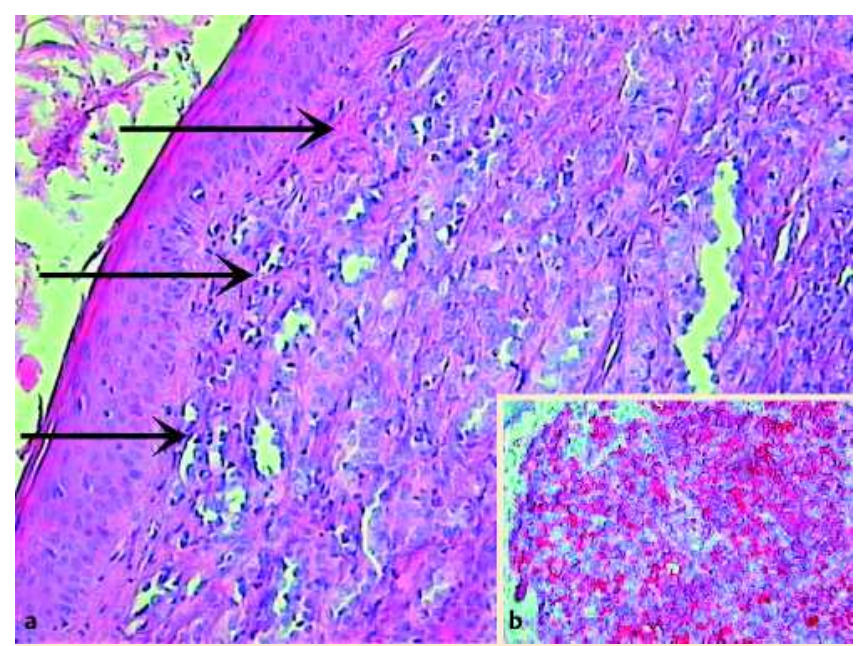

Abb. 1 Histologische (a) und immunhistochemische (b) Untersuchungen eines kutanen Tumorknotens mit a) lichtmikroskopischem Nachweis eines epitheloiden Tumors (Pfeile) (HE-Färbung, 100-fach) und b) positiver Immunreaktion gegen CD31 (rot) (200-fach) als Nachweis der angiogenen Differenzierung.

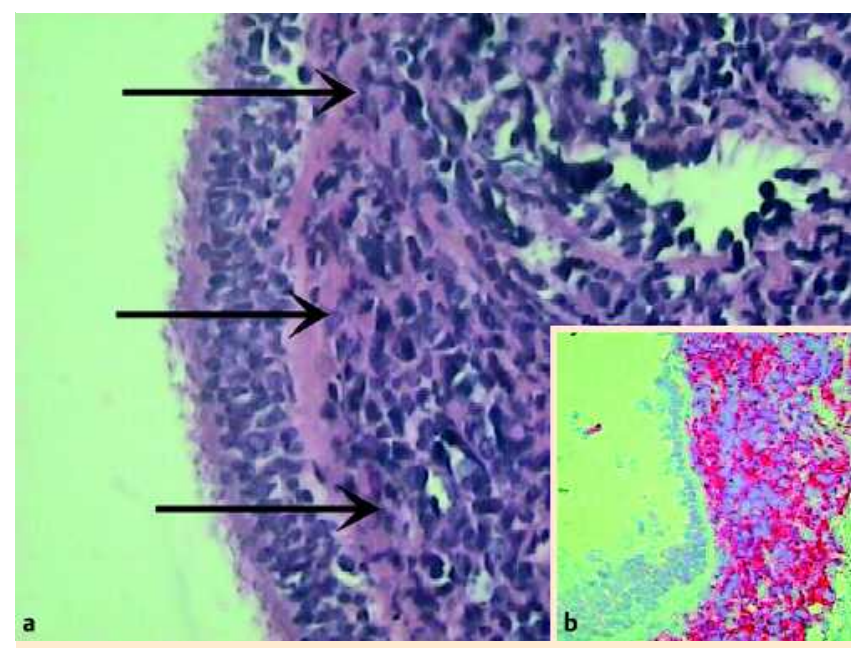

Abb. 2 Histologische (a) und immunhistochemische (b) Untersuchungen einer Bronchialschleimhautbiopsie mit a) lichtmikroskopischem Nachweis eines klein-, rund- und blauzelligen Tumors (Pfeile) (HE-Färbung, 200-fach) und b) positiver Immunreaktion gegen CD31 (rot) (100-fach) als Nachweis der angiogenen Differenzierung.

tasen in den Nieren und im perirenalen Fettgewebe sowie eine Metastase in der Herzhinterwand nachweisbar.

Ein Zufallsbefund war ein 4,2 cm großes, klarzelliges Nierenzellkarzinom rechts. Histologisch und immunhistologisch ließ sich eine Metastasierung des Angiosarkoms in das Nierenzellkarzinom darstellen. Die Zellen des Nierenzellkarzinoms reagierten mit Antikörpern gegen CD10 eindeutig positiv. Mit Antikörpern gegen CD31 zeigte sich keine Reaktion. Die Zellen des Angiosarkoms waren CD10 komplett negativ und CD31 kräftig positiv. Die abschließende Beurteilung ergab somit ein lymphogen und hämatogen metastasierendes Angiosarkom der Haut bei $\mathrm{Zu}-$ stand nach Operation eines Ovarialkarzinoms mit postoperativer Radiatio vor 27 Jahren. 


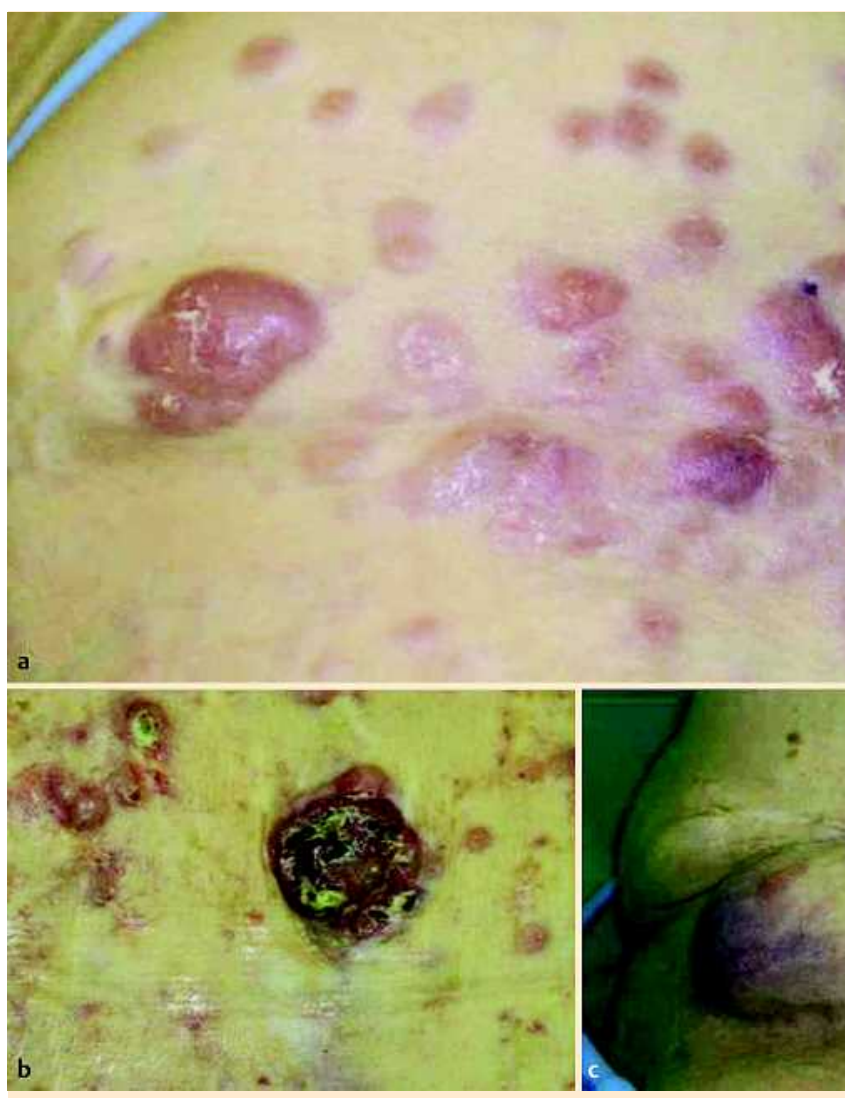

Abb. 3 a-c Makroskopischer Aspekt der kutanen Manifestationen des Angiosarkoms in der Bauchdecke (Sektionsbefund).

\section{Diskussion}

$\nabla$

Die Differenzialdiagnose interstitieller, lymphozytenähnlicher Infiltrate in der Lunge schließt neben entzündlichen Veränderungen wie der lymphozytären interstitiellen Pneumonitis (LIP) und der exogen-allergischen Alveolitis auch die breite differenzialdiagnostische Palette der malignen klein-, rund- und blauzelligen Tumoren ( $\odot$ Tab. 1$)$ ein [14-16]. In der Lunge kommen davon in erster Linie ein kleinzellig anaplastisches Karzinom und ein Lymphom in Betracht. Die Differenzierung erfordert
Tab. 1 Differenzialdiagnosen maligner klein-, rund- und blauzelliger Tumoren.

kleinzelliges anaplastisches Karzinom (Kleinzell. Ca.)
niedrig malignes Non-Hodgkin-Lymphom (NHL-NM)
Alveoläres Rhabdomyosarkom (RMS)
Embryonales Rhabdomyosarkom (RMS)
Desmoplastischer maligner kleinzelliger Tumor der Kindheit
Extraskeletales Ewingssarkom
Neuroblastom
Primitiver neuroektodermaler Tumor (PNET)
Liposarkom - rundzellige Variante
Mesenchymales Chondrosarkom
Malignes Hämangioperizytom
Leiomyosarkom
Angiosarkom
Osteosarkom
Malignes fibröses Histiozytom (MFH)
Solitärer fibröser Tumor (SFT)
Mesotheliom
Merkelzell-Karzinom (Merkelzell-Ca.)

eine systematische immunzytologische bzw. immunhistologische Aufarbeitung ( $\bullet$ Tab. 2) $[14,16]$.

Das Angiosarkom ist eine seltene Neoplasie des Weichgewebes. Der Altersgipfel liegt im siebenten Lebensjahrzehnt. Die häufigsten Lokalisationen sind die Muskulatur der Extremitäten sowie die Bauchhöhle [1 -3].

Radiogen induzierte Angiosarkome sind sehr selten. Bisher existieren hierzu nur kleine Studien und Einzelfallberichte [1,11]. Bei unserer Patientin besteht aufgrund des plausiblen zeitlichen Intervalls und der strengen Lokalisation im ehemaligen Bestrahlungsgebiet kein begründeter Zweifel an der aktinischen Genese des primär kutanen Angiosarkoms.

Angiosarkome lassen ein charakteristisches immunhistochemisches Muster erkennen, was die differenzialdiagnostische Abgrenzung von anderen klein-, rund- und blauzelligen Tumorentitäten ermöglicht ( $\odot$ Tab.2). Bei immunhistologischen Untersuchungen zeigen sie eine Reaktion mit Antikörpern gegen CD31 (90\%), den von-Willebrand-Faktor und CD34.

Die Prognose und das Ansprechen auf eine zytostatische Therapie sind bei dieser Tumorentität sehr schlecht. Das Angiosarkom ist ein sehr aggressiver Tumor mit einer hohen Lokalrezidivrate und

Tab. 2 Immunhistologisches Profil maligner klein-, rund- und blauzelliger Tumoren (Ca.: Karzinom; PNET: Primitiver neuroektodermaler Tumor; MFH: Malignes fibröses Histiozytom, SFT: Solitärer fibröser Tumor, RMS: Rhabdomyosarkom, CK: Cytokeratin, CK MNF 116: Cytokeratin Klon 116, TTF1: thyreoidaler Transkriptionsfaktor 1, CD: cluster of differentiation).

\begin{tabular}{|c|c|c|c|c|c|c|c|c|c|c|}
\hline & CK MNF116 & CK20 & CD56 & TTF1 & $\begin{array}{l}\text { Chromo- } \\
\text { granin }\end{array}$ & $\begin{array}{l}\text { Synapto- } \\
\text { physin }\end{array}$ & CD45 & Vimentin & CD99 & CD34 \\
\hline Kleinzell. Ca. & + & - & + & + & + & + & - & - & - & - \\
\hline Lymphom * & - & - & - & - & - & - & + & + & - & - \\
\hline Leiomyosarkom * & - & - & - & - & - & - & - & + & - & - \\
\hline Angiosarkom & $+1-$ & - & - & - & - & - & - & + & - & $+1-$ \\
\hline RMS * & - & - & - & - & - & - & - & + & - & - \\
\hline Osteosarkom * & - & - & - & - & - & - & - & + & - & - \\
\hline PNET & - & - & - & - & - & - & - & + & + & - \\
\hline MFH & - & - & - & - & - & - & - & - & - & - \\
\hline SFT & - & - & - & - & - & - & - & + & - & + \\
\hline Mesotheliom * & + & - & - & - & - & - & - & + & - & - \\
\hline Merkelzell-Ca. & + & + & + & - & + & + & - & - & - & - \\
\hline
\end{tabular}

* weitere immunhistologische Untersuchungen zur endgültigen Differenzierung. 
einer frühzeitigen Metastasierung in die Lungen, die Lymphknoten, die Knochen und die Weichgewebe $[1-3,10,11,17]$.

Das bei unserer Patientin vorliegende, eine interstitielle Lungenerkrankung imitierende Metastasierungsmuster war durch ein vorwiegend interstitielles und peribronchiales Tumorwachstum bedingt. Ein derartiges Metastasierungsmuster ist äußerst ungewöhnlich und erfordert eine breite Differenzialdiagnose, die einen frühzeitigen Einsatz endoskopisch-bioptischer Verfahren und eine enge Kommunikation zwischen Kliniker und Pathologen erfordert.

Die Metastasierung eines Angiosarkoms in ein synchrones Nierenzellkarzinom, wenngleich für die Patientin ohne klinische Relevanz, wurde bisher nicht beschrieben. Wir vermuten, dass die gute Durchblutung des Nierenzellkarzinoms infolge der Tumorneovaskularisation die Metastasierung begünstigt hat. So wurden Metastasen anderer maligner Tumoren in Nierenzelltumoren bereits mehrfach kasuistisch beschrieben $[18,19]$.

\section{Abstract}

\section{Unusual Metastatic Pattern of a Radiation Induced Angiosarcoma of the Skin \\ $\nabla$}

We report on a 67 year old female patient in whom hysterectomy and oophorectomy as well as adjuvant telecobalt radiation were performed 27 years ago. 25 years later, multiple tumours occurred in the abdominal wall. Histological examination of these tumours showed an epitheloid neoplasm with vascular proliferation and a fine framework of reticular fibres. Immunohistochemistry revealed angiogenic differentiation and an angiosarcoma was diagnosed. It was treated surgically.

Another two years later the patient was admitted to hospital with severe dyspnoea. Clinical examination showed multiple reddish nodules in the abdominal wall. Chest $\mathrm{x}$-ray and CT-scans showed abundant bilateral predominantly interstitial infiltrates in both lungs. Bronchoscopy revealed a diffuse thickening of the bronchial mucosa. Bronchoalveolar lavage, biopsies of the bronchial mucosa and transbronchial lung biopsies were performed. A malignant small round blue cell tumour was identified in all specimens. Immunohistochemistry confirmed metastases of the angiosarcoma.

Post-mortem examination revealed relapse of the angiosarcoma in the abdominal wall as well as metastases in the lungs, which showed a predominantly interstitial and peribronchial growth pattern. Further metastases were found in the lymph nodes, in the myocardium, in the abdominal fatty tissue and additionally in a synchronous renal cell carcinoma.

Radiation induced angiosarcoma is very uncommon. Furthermore, the metastatic pattern with the clinical presentation as an interstitial lung disease on the one hand and with a metastasis in another malignant tumour, which occurred independently, on the other hand is an extreme rarity.

\section{Literatur}

1 Weiss SW, Goldblum JR. Enzinger and Weiss's Soft Tissue Tumors. 4th edition. London: Mosby, 2001

2 Fletcher CDM, Unni KK, Mertens F. World Health Organization Classification of Tumours. Pathology and Genetics of Tumours of Soft Tissue and Bone. Lyon: IARC Press, 2002

3 Kempson RL, Fletcher CDM, Evans HL, Hendrickson MR, Sibley RK. Atlas of Tumor Pathology. Tumors of the Soft Tissues. Third series. Fascicle 30. Washington, DC: Armed Forces Institute of Pathology, 2001

4 Kauffmann GW, Moser E, Sauer R. Radiologie. 2. Auflage. München, Jena: Urban \& Fischer, 2001

5 Salvati M, D'Elia A, Melone GA, Brogna C, Frati C, Raco A, Delfini R. Radio-induced gliomas: 20-year experience and critical review of the pathology. J Neurooncol 2008; Jun 20 (Epub ahead of print)

6 Gessi M, Maderna E, Guzette S, Cefalo G, Massimino M, Solero CL, Finocchiaro $G$, Pollo $B$. Radiation-induced glioblastoma in a medulloblastoma patient: a case report with molecular features. Neuropathology 2008; Apr 1 (Epub ahead of print)

7 Brockstein B, Mundt A, Haraf DJ, Ferguson M, Montag A. Radiation-induced leiomyosarcoma: does antimetabolite chemotherapy contribute? A report of three cases. Sarcoma 2003; 7: 167-172

8 Noh JM, Huh SJ. Two cases of post-radiation osteosarcoma of the sacrum after pelvic irradiation for uterine cervical cancer. Eur J Gynaecol Oncol 2007; 28: 497-500

9 Vuong NT, Boucher E, Gedouin D, Vauleon E, LePrise E, Raoul JL. Radiation-induced oesophageal carcinoma after breast carcinoma: a report of five cases including three successfully treated by radiochemotherapy. Acta Oncol 2007; 46: $1184-1186$

10 LeBoit PE, Burg G, Weedon D, Sarasin A. World Health Organization Classification of Tumours. Pathology and Genetics of Skin Tumours. Lyon: IARC Press, 2006

11 Weedon D. Skin Pathology. 1st edition. New York: Churchill Livingston Inc, 1997

12 Patton KT, Deyrup AT, Weiss SW. Atypical vascular lesions after surgery and radiation of the breast: a clinicopathologic study of 32 cases analyzing histologic heterogeneity and association with angiosarcoma. Am J Surg Pathol 2008; 32: 943 - 950

13 Holloway CL, Turner AR, Dundas GS. Cutaneous angiosarcoma of the scalp: a case report of sustained complete response following liposomal Doxorubicin and radiation therapy. Sarcoma 2005; 9: 29-31

14 Travis WD, Brambilla E, Müller-Hermelink HK, Harris CC. World Health Organization Classification of Tumours. Pathology and Genetics of Tumours of the Lung, Pleura, Thymus and Heart. Lyon: IARC Press, 2004

15 Travis WD, Colby TV, Koss MN. Atlas on Nontumor Pathology. NonNeoplastic Disorders of the Lower Respiratory Tract. First Series. Washington, DC: Armed Forces Institute of Pathology, 2002

16 Lüdike A, Knolle J, Schön R, Hinze P, Hofmann HS, Schreiber J. Primary pulmonary rhabdomyosarcoma as a rare differential diagnosis of small cell lung cancer. Pneumologie 2005; 59: 456-460

17 Murphy GF, Elder DE. Atlas of Tumor Pathology. Non-Melanocytic Tumors of the Skin. Third series. Fascicle 1. Washington, DC: Armed Forces Institute of Pathology, 1991

18 Ricketts R, Tamboli P, Czerniak B, Guo CC. Tumor-to-Tumor Metastasis, Report of 2 Cases of Metastatic Carcinoma to Angiomyolipoma of the Kidney. Arch Pathol Lab Med 2008; 132: 1016 - 1020

19 Sella A, Ro JY. Renal cell cancer: best recipient of tumor-to-tumor metastasis. Urology 1987; 30: 35-38 